\title{
Planificación de parques urbanos: la experiencia estadounidense
}

\section{Planning of urban parks: the American experience}

\author{
Harnik, Peter (20io), Urban Green: Innovative Parks for Resur- \\ gent Cities, Island Press, Washington, i 84 PP., isbn: I 3:978-I-59- \\ 726-684-O, ISBN: IO:I.59726-684-I.
}

La publicación de este libro parte del interés que tiene Peter Harnik en la planificación y diseño de parques urbanos en Estados Unidos y que se refleja en sus actividades como asesor e investigador en las áreas de planificación, diseño, conservación y mantenimiento de parques públicos; además de ser un entusiasta activista a favor de los espacios verdes. Peter Harnik es director del Civil Center for City Park Excellence Staff, que pertenece a The Trust for the Public Land. Esta última es una organización civil sin fines de lucro, cuyo objetivo fundamental es incentivar la conservación de áreas que se puedan destinar a parques, jardines comunitarios y áreas naturales a lo largo y ancho de Estados Unidos.

En el libro Inside City Parks (2000), Harnik hizo un primer acercamiento al tema de planificación de sistemas verdes urbanos, empleando estadísticas estadounidenses sobre parques urbanos y recreación, como una herramienta de apoyo en la toma de decisiones de agentes gubernamentales y especialistas en espacios urbanos. En dicho libro, parte de la comparación estadística de cifras de inventarios de parques (número, superficies, programas, densidades) entre ciudades, para identificar cómo los intereses públicos y privados influyen en la planificación y desarrollo de sistemas de parques citadinos. Sin embargo, es en el libro que reseñamos donde mediante el empleo de estadísticas nacionales similares, acota la discusión. El libro consta de 26 capítulos divididos en dos partes (I. "Of Cities and Parks", II. "Finding Park"), en las cuales especifica cuatro temas que son de interés en el área de planificación de sistemas de áreas verdes y parques citadinos:

a) La utilidad de indicadores de inventario a los cuales denomina cifras mágicas para quienes toman las decisiones.

b) La importancia fundamental de conocer las necesidades, gustos y preferencias de la gente que harán uso de los espacios verdes en las ciudades. 
c) Una propuesta de tipología de espacios verdes urbanos, basados en las necesidades de los usuarios más que en los intereses particulares de planificadores/agentes gubernamentales y/o perspectivas de diseño que tengan ingenieros y arquitectos.

d) Los temas anteriores establecen un cambio de perspectiva en el diseño y planificación de sistemas verdes, lo cual lleva a considerar formas potenciales a través de las cuales las ciudades estadounidenses pueden hacerse de nuevos espacios verdes públicos, lo que se describen en la parte II del libro.

Harnik utiliza estadísticas que permiten comparar cifras de inventario verde en ciudades estadounidenses, particularmente de superficies de parques, densidad (personas por acre de parque) y acres de parque por 1,000 habitantes; las estadísticas se presentan considerando una clasificación de ciudades de acuerdo con un indicador de densidad, dividiéndolas en ciudades de alta, mediana y baja densidad. Igualmente, refiere al lector a la página de Internet de The Trust for the Public Land (2010), donde se pueden consultar cifras de inventario de número de parques por ciudad, entre otras estadísticas, informes de investigación y documentos de análisis de la situación actual de los parques urbanos estadounidenses. Dichos trabajos son producto del esfuerzo conjunto entre investigadores de The Trust for the Public Land y otros especialistas en el tema de parques urbanos, entre los que se incluyen arquitectos, economistas y ecólogos, entre otros. En los primeros capítulos del libro, Harnik hace uso de las estadísticas señaladas e invita al lector a consultar dichas cifras, no con la finalidad de determinar qué ciudad tiene más superficie de parques sino con el objetivo de que el lector observe los fallos o sesgos en los que el planificador, agente o ciudadano puede incurrir al comparar diferencias de dotación en ciudades con densidades diferentes.

Con base en esto, Harnik hace una crítica directa a las cifras mágicas que diversas agencias internacionales establecen como indicadores óptimos de áreas verdes urbanas, que aunque no menciona directamente, sugiere el uso aislado (excluyendo criterios de necesidades y gustos sociales) por parte de agentes gubernamentales y planificadores, es decir los $9 \mathrm{~m}^{2}$ per cápita que se manejan y el tiempo de acceso de cada citadino a un espacio verde: no más de 15 minutos a pie sugeridos por la Organización Mundial de la Salud y diversos especialistas en planeación (Sorensen et al., 1998; Salvador, 2003). Asimismo, el autor lleva a reflexionar sobre el uso excesivo y/o aislado que se hace de tales indicadores mágicos y, aunque no aporta más evidencia sobre el fallo de tales indicadores, sugiere como primer indicador de planificación el conocimiento y valoración de las necesidades, gustos y preferencias de los usuarios como piedra nodal en 
el diseño de nuevos espacios o bien en el desarrollo de programas de mejora o recuperación de espacios verdes existentes. A fin de cuentas, Harnik argumenta que son los usuarios quienes debieran expresar sus necesidades y requerimientos, por lo que es la conciencia social-ambiental de los citadinos y las organizaciones sociales quienes deberían ejercer el poder necesario para presionar a los gobiernos locales en favor de los parques urbanos. De acuerdo con el autor, hablar de planificación de áreas verdes y parques urbanos es hablar de lo que la gente quiere, mas no de lo que se debe tener. Consecuentemente, el que una ciudad tenga áreas verdes es más un problema de conciencia ambiental y presión política que un problema de planificación urbana de escritorio y planos que considere fríamente un indicador de superficie que se debe alcanzar de acuerdo con normas internacionales. Y aún más, Harnik sugiere que la atención de gustos y necesidades debiera incluso alejarse un poco de la clasificación vetusta y complicada de gustos recreativos: activa frente a pasiva, bajo la cual todavía se siguen diseñando programas de atención a visitantes de parques. Para esto sugiere una nueva tipología de parques basadas en las necesidades de los usuarios y no en las frías tipologías urbanistas encaminadas a cubrir estándares de dotación. Además destaca que la mayoría de las ciudades ya están desarrolladas, por lo que su objetivo es encontrar superficie para incorporar a un sistema que cubra necesidades diversas más que la conservación de espacios verdes per se. El autor sugiere una tipología desarrollada por planificadores de la ciudad de Portland, Oregon, que permite sin complicaciones abarcar una diversidad de actividades o intereses que pueden comprender los parques urbanos. Esta clasificación consta de las siguientes posibilidades:

a) Gente a gente: incluye actividades donde la socialización es necesaria, como deportes, actividades familiares, entre otras.

b) Gente a naturaleza: consta de actividades como contemplar el paisaje, observación de vida silvestre, etcétera.

c) Naturaleza a naturaleza: la cual comprende únicamente actividades de conservación de espacios verdes.

Igualmente aclara que los espacios pueden incluir una o más de las actividades que sugiere esta clasificación

Con esta visión, Harnik invita indirectamente al lector a revalorar toda la cauda de estudios de gustos, preferencias y modelos de utilidad recreativa en parques urbanos, como los elaborados por Payne et al. (2002), Sasidharan et al. (2005) y Kemperman y Timmermans (2006), los cuales cobran vital importancia desde la perspectiva propuesta por Harnik. Hay que destacar que la importancia de considerar los gustos y preferencias 
que tienen los citadinos por sus áreas verdes también ha sido considerada por otros autores: en Europa DeVos (2005) y Falcón (2007), en Argentina Tella et al. (2007) y en México Flores y González (2007). Estos autores han señalado la necesidad de incorporar criterios sociales a la planificación verde, a partir de la evaluación de necesidades, gustos y preferencias. Igualmente Harnik no es el primero en cuestionar la utilidad y sustento de los indicadores de dotación, en Latinoamérica, Gámez (2005) y Flores y González (2010) han analizado y discutido la utilidad de los indicadores de dotación de superficie en los programas de planificación de sistemas de áreas verdes citadinas. Particularmente, Flores y González (2010) sugieren que los indicadores propuestos por agencias como la Organización Mundial de la Salud ( $9 \mathrm{~m}^{2} /$ per cápita y de tiempo de acceso: no más de 15 minutos del lugar de residencia), los deberían evaluar e investigar especialistas, tomando como referencia las características ambientales, históricas, sociales y geográficas de las urbes. En consecuencia, los argumentos y recomendaciones de Peter Harnik y de los autores mencionados, incentivan el desarrollo de futuras investigaciones que permitan determinar qué tan útiles son los indicadores de superficie y tiempo de acceso, además de la importancia de generar un proceso de toma de decisiones en planeación de sistemas verdes combinando criterios económicos, sociales y ecológicos. Además, Harnik omite mencionar la necesidad de revitalizar los parques urbanos considerando la diversidad social, la cual está presente en muchas ciudades estadounidenses y que ha sido tema de estudio de investigadores como Low et al. (2005), y que en Europa ha llevado a sugerir estrategias de planificación incluyente y de manejo por áreas a través de consensos sociales (DeVos, 2005), lo cual ha permitido incluir en el diseño de los espacios verdes a grupos sociales históricamente olvidados, excluidos y acosados en espacios verdes, como jóvenes, etnias, personas transgénero, homosexuales, personas con capacidades diferentes, entre otros (Flores y González, 2007). Este hecho cobra particular importancia en ciudades de naciones emergentes, como las latinoamericanas, donde los procesos participativos sociales dirigidos a conocer e incorporar demandas sociales no se consideran en el diseño de estrategias para hacer más verde la planificación urbana.

Por último, en la segunda parte de su libro, Harnik describe estrategias novedosas mediante las cuales diversas ciudades estadounidenses se han hecho de parques urbanos. Algunas de estas estrategias son:

a) Apertura de áreas verdes pertenecientes a escuelas durante los fines de semana o en horas fuera de horarios de clase.

b) Apertura de áreas verdes en cementerios. 
c) Diseño de jardines comunitarios donde se pueden realizar actividades de horticultura y de agricultura urbana.

d) Techos verdes.

No obstante, Harnik no pone el mismo énfasis en señalar que posiblemente el diseño de algunos de estos espacios pudieran tener efectos favorables en provisión de servicios ambientales (captura de carbono, provisión de oxígeno, entre otros), y que, por otro lado, las utilidades de esos espacios sociales recreativos serían restringidas.

De esta forma, el libro tiene un interés especial para todos aquellos que estamos interesados en la planificación de áreas verdes y parques urbanos, trátese de activistas, investigadores, consultores o funcionarios gubernamentales, ya que los argumentos señalados por Harnik se podrían incorporar a nuevos proyectos de investigación, proyectos arquitectónicos de diseño, planes de desarrollo urbano y en el diseño, implementación y evaluación de políticas gubernamentales a favor de la recuperación de espacios públicos citadinos, como el que está realizando el gobierno federal mexicano (Sedesol, 2010).

\section{Bibliografía}

DeVos, Els (2005), "Public Parks in Ghent's City Life: From Expression to Emancipation?", European Planning Studies, 13 (7), Londres, pp. 1035-1061.

Falcón, Antoni (2007), Espacios verdes para una ciudad sostenible, Gustavo Gilli, Barcelona.

Flores-Xolocotzi, Ramiro y Manuel González-Guillén (2007), "Consideraciones sociales en el diseño y planificación de parques urbanos", Economía, Sociedad y Territorio, 6 (24), El Colegio Mexiquense, Zinacantepec, pp. 913-951.

Flores-Xolocotzi, Ramiro y Manuel de Jesús González-Guillén (2010), "Planificación de sistemas de áreas verdes y parques públicos de algunas ciudades del mundo", Revista Mexicana de Ciencias Forestales, 35 (107), México (en prensa).

Gámez, Vicente (2005), "Sobre sistemas, tipologías y estándares de áreas verdes en el planeamiento urbano", Diseño Urbano y Paisaje, 6, Santiago de Chile, pp. 1-22. 
Kemperman, Astrid y Harry Timmermans (2006), "Heterogeneity in urban parks use of aging visitors: a latent class analysis", Leisure Sciences, 28 (1), Filadelfia, pp. 57-71.

Low-Setha, Dana Taplin y Suzanne Scheld (2005), Rethinking Urban Parks Public Space and Cultural Diversity, The University of Texas Press, Texas.

Payne-Laura, Andrew Mowen y Elizabeth Orsega-Smith (2002), "An examination of park preferences and behaviors among urban residents: the role of residential location, race and age", Leisure Sciences, 24 (1), Filadelfia, pp. 181-198.

Salvador, Pedro (2003), La planificación verde en las ciudades, Gustavo Gilli, Barcelona.

Sasidharan, Vinod, Fern Willits y Geoffrey Godbey (2005), "Cultural differences in urban recreation patterns: an examination of park usage and activity participation across six population subgroups", Managing Leisure, 10 (1), Londres, pp. 19-38.

Sedesol (Secretaría de Desarrollo Social) (2010), Lineamientos especificos para la operación del Programa de Rescate de Espacios Públicos, Sedesol, México.

Sorensen, Mark, Valerie Barzetti, Kari Keipi y John Williams (1998), Manejo de las áreas verdes urbanas. Documento de buenas prácticas, Banco Interamericano de Desarrollo, Washington.

Tella, Guillermo, Estela Cañellas, Viviana Colella, Luciana Garavaglia y Daniela Natale (2007), "La emergencia del parque social. Expresiones del proceso de contraurbanización en Buenos Aires", Espiral, 13 (38), Guadalajara, pp. 141-168.

The Trust for the Public Land (2010), News/publications <http://www. tpl.org/tier2_rp1.cfm?folder_id=174>, 15 de noviembre de 2010.

Recibida: 29 de noviembre de 2010. Aceptada: 5 enero de 2011. 
Ramiro Flores-Xolocotzi

El Colegio de Tlaxcala, A.c. Correo-e: pinos42@hotmail.com

Ramiro Flores-Xolocotzi. Es profesor-investigador del Colegio de Tlaxcala, A.c. Hizo un posdoctorado en planeación de áreas verdes urbanas en el Programa de Estudios Urbanos y Ambientales de El Colegio Mexiquense; es doctor en ciencias forestales con especialidad en economía ambiental por el Colegio de Postgraduados. Sus líneas de investigación son: planificación de áreas verdes urbanas y economía ambiental. Entre sus publicaciones sobresalen: en coautoría, "Consideraciones sociales en el diseño y planificación de parques urbanos", Economía, Sociedad y Territorio, 6 (24), El Colegio Mexiquense, Zinacantepec, pp. 913-951 (2007); en coautoría, "Planificación de sistemas de áreas verdes y parques públicos de algunas ciudades del mundo", Revista Mexicana de Ciencias Forestales, 35 (107), México (2010, en prensa); en coautoría, "Valoración económica del servicio recreativo del Parque Hundido de la Ciudad de México", Región y Sociedad, 22 (47), Hermosillo, pp. 123-144 (2010). 